\title{
Surface Roughness Instabilities in Low-Angle Ion Milling
}

\author{
A. W. Barnard*, J. K. Hyun*, J. L. Grazul**, D. A. Muller*,** \\ *School of Applied and Engineering Physics, Cornell University, Ithaca, NY 14853 \\ **Cornell Center for Materials Research, Cornell University, Ithaca, NY 14853
}

The ion-beam thinning of a sample involves a general competition between sputtering, implantation, and surface migration of atoms. The result is a complicated phase space that contains self-organized patterns, roughening transitions and (hopefully) a regime of smooth erosion with limited intermixing[1-4]. At normal incidence the momentum transfer is largely in a forward direction, into the target removing relatively little material. At grazing incidence the sputtering rate is again low (actually tending to zero) as the beam is spread over an almost infinitely large projected area. Somewhere between these two extremes, the sputtering rate must reach a maximum value. For Si (001) this is usually found near 25 degrees from grazing incidence[1] (we will reference all angles from grazing incidence). Milling near this local maximum can lead to roughening instabilities and amplification of existing defects. If macrosteps are allowed to migrate along the surface as they are eroded away, a mechanism for smoothing is introduced. The step migration rate is inversely proportional to the sputtering rate, so near the sputtering maximum little smoothing can occur[1,2]. These are universal features that qualitatively control the surface-roughness phase diagram[2], providing important guidance for optimizing both $\mathrm{Ar}$ and Ga-ions processes.

It has generally been thought that for angles well below $25^{\circ}$, Ar-ion milling should smooth the surface. However, with new mills operating at lower angles and lower voltages, this has not always been the case and effects of larger beam divergence and wider momentum spread may cause unwanted instabilities. To gain a more empirical understanding of roughening characteristics for standard milling parameters, we undertook a systematic characterization of surface morphology using atomic force microscopy for initially flat silicon crystals milled at different incident angles and beam voltages (Fig 1).

Si (100) samples were prepared by chemical cleaning and mounted on $3 \mathrm{~mm}$ grids. After curing for 24 hours, samples were milled in a Fischione Model 1010 Ion mill, using typical milling parameters: an in-plane rocking angle of $\pm 40^{\circ}$, a beam current of $5 \mathrm{~mA}$, and a milling time of 30 minutes. The milling angle was varied in a range of 8-25 degrees from grazing incidence and beam voltage was varied. ( $2 \mathrm{kV}$ beam voltage is shown in Fig 1). Milled samples were then viewed with a Dimension 3100 AFM operating in tapping-mode with Olympus Si cantilevers. For consistency, images were taken at the center of samples with scan sizes of $5 \mu \mathrm{m}$ and $2.33 \mu \mathrm{m}$. Finally, using Nanoscope v5.4b1, root-mean square (RMS) roughness was calculated.

Figure 1 (a) shows a sample milled at a relatively low angle of $10^{\circ}$, the resulting RMS roughness of .162nm is well within the range desired for a polished XTEM (cross-section TEM) sample, and comparable to the initial starting roughness. Low RMS roughness persists up to $12^{\circ}$, with a slight increase in roughness with increasing beam angle. There is a dramatic and qualitative change in surface roughness between $12^{\circ}$ and $13^{\circ}$ as seen in Figure 1 (d) which appears mark the transition between the smooth and rough growth regimes. We note this is half the angle expected from earlier work[1]. Above this angle, the roughness decreases, presumably as we move away 
from the angle of maximum sputtering rate. If the beam current is fixed, then as the beam voltage is reduced for final polishing, we would also expect the beam divergence to increase, causing the roughnening instability to set in at an even-lower milling angle.

[1] A. Barna, P. B. Barna, Vacuum 40, 115 (1990).

[2] R. Cuerno, A.-L. Barabasi, Phys. Rev. Lett. 74, 4746 (1995).

[3] F. Frost, et al., Nucl. Instr. Meth. in Phys. B216, 9 (2004).

[4] R. Gago, et al., Appl. Phys. Lett. 78, 3316 (2001).

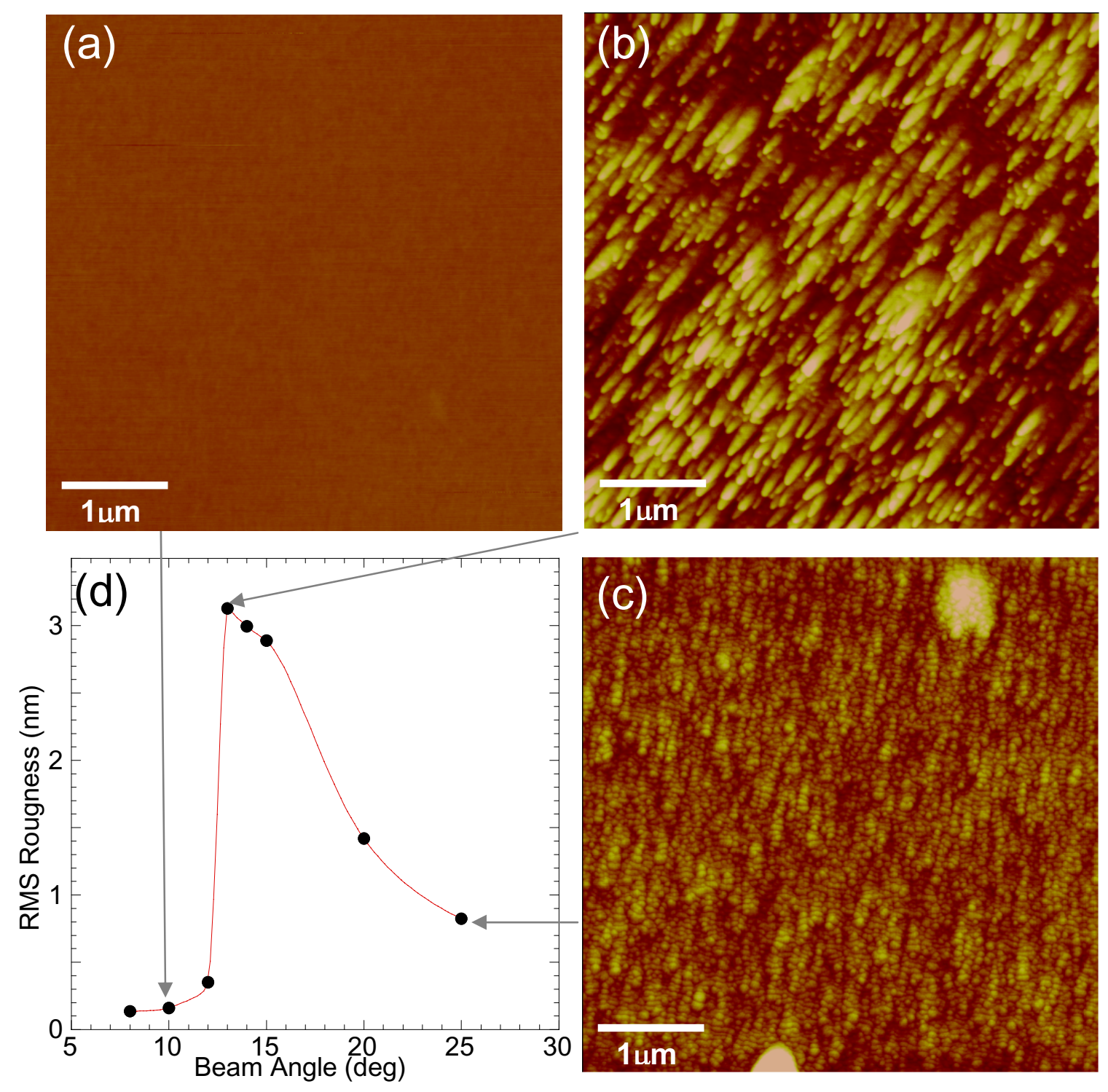

FIG 1. Atomic Force Microscopy of three (001) silicon surfaces ion milled with $2 \mathrm{keV}$ Ar ions (5 $\mathrm{mA}$ beam current, $\pm 40^{\circ}$ sample rotation) for 30 minutes at (a) $10^{\circ}$ (b) $13^{\circ}$ (c) $25^{\circ}$ to the plane of the surface. (d) Summary of the root-mean-square surface roughness as a function of incident beam angle showing a roughening instability near $13^{\circ}$. 